\title{
Fragility of Bioprotectant Glass-Forming Systems in Extremophiles
}

\author{
S. Magazù̀ ${ }^{1}$ and F. Migliardo ${ }^{1,2}$ \\ ${ }^{1}$ Department of Physics, University of Messina, P.O. Box 55, Sperone 31, 98166 Messina, Italy \\ ${ }^{2}$ Laboratoire de Dynamique et Structure des Matériaux Moléculaires, University of Lille 1, \\ CNRS UMR 8024, 59655 Villeneuve d'Ascq Cedex, France
}

Correspondence should be addressed to F. Migliardo, fmigliardo@unime.it

Received 25 January 2009; Revised 20 March 2009; Accepted 23 March 2009

Recommended by Derrick S. F. Crothers

\begin{abstract}
A central issue in the adaptation of proteins and enzymes to extreme conditions is the conservation of their functional state, which is characterized by a well-balanced compromise of stability and flexibility. In this review work an overview of elastic neutron scattering (ENS) findings on a class of bioprotectant glass-forming systems, such as trehalose and its homologous (maltose and sucrose) water mixtures, is presented as a function of temperature and concentration. ENS, in fact, allows to determine some remarkable quantities in order to characterize the correlation among dynamical properties, the flexibility and fragility of biomolecules. The experimental results have pointed out a dynamical transition, which shows a crossover in molecular fluctuations between harmonic and anharmonic dynamical regimes. The ENS findings allow to characterize both the trehalose rigidity and flexibility, which are strictly connected to its superior bioprotective effectiveness. In this frame the lowest flexibility and fragility character of trehalose $/ \mathrm{H}_{2} \mathrm{O}$ mixture with respect to maltose and sucrose $/ \mathrm{H}_{2} \mathrm{O}$ mixtures indicate a better attitude to encapsulate biostructures in more rigid and temperature insensitive structures in approaching the glass transition.
\end{abstract}

Copyright (C) 2009 S. Magazù and F. Migliardo. This is an open access article distributed under the Creative Commons Attribution License, which permits unrestricted use, distribution, and reproduction in any medium, provided the original work is properly cited.

\section{Introduction}

In recent years a lot of attention has been addressed to the understanding of the mechanisms present in organisms able to survive under environmental stress conditions, that is, the extremophiles [1-10].

Trehalose is a glass-forming nonreducing disaccharide of glucose widely distributed in both prokaryotic and eukaryotic organisms that, very surprisingly, show the ability of adaptation to extreme environmental stress conditions [11-20]. Thanks to trehalose, many living beings capable of surviving extreme conditions enter into a state of suspended animation, called "cryptobiosis" [21]. Cryptobiosis, from Greek $\kappa \rho v \pi \tau o \varsigma$, "hidden" and "concealed", and $\beta \iota \sigma \sigma$, "life", refers to a particular state of organism inactivating when prohibitive environmental conditions occur.

Four cryptobiotic pathways, anhydrobiosis, cryobiosis, osmobiosis, and anoxybiosis, exist in nature. Anhydrobiosis, a form of cryptobiosis initiated by desiccation, is an almost complete loss of body water and allows organisms to stay in this state for an extended period of time [22]. Cryobiosis, initiated by a reduction of temperature, involves the ordered freezing of water within the cells and allows organisms to tolerate rapid freezing [23]. Osmobiosis is determined by a decreased water potential due to increased solute concentration in the surrounding solution, while anoxybiosis indicates a reduction of oxygen tension $[21,24]$.

To highlight the ubiquity and extraordinary bioprotective effectiveness of trehalose, it should be noticed that the disaccharide has been found in all the natural kingdoms. One of the most significant examples of the biological role of trehalose is represented by tardigrada, that, thanks to the disaccharide synthesis, are able to survive at temperatures near absolute zero and above the boiling point of water, pressure of $6000 \mathrm{~atm}$, a hard vacuum (as in outer space), high doses of radiation, X-rays, nitric acid, hydrochloric acid, carbon dioxide, and carbon monoxide. They can live more 125 years, alternating periods of active life and of cryptobiotic life [25]. 
Trehalose has been found also in plants. Arabidopsis thaliana, a small plant in the mustard family, has recently been demonstrated to possess genes capable of complementing yeast mutants defective in the two steps of trehalose biosynthesis [26]. The "resurrection plants" found in the desert are a particular example of plants surviving in arid environments because they are able to dehydrate, remain quiescent during long periods of drought, and then resurrect upon rehydration. The desert pteridophyte Selaginella lepidophylla [27] represents a model to study the ecology, physiology, biochemistry, and photochemistry of extreme desiccation tolerance. Dehydration induces the expression of a large number of transcripts in resurrection plants: gene products with a putative protective function, such as LEA proteins, are expressed at high levels in the cytoplasm or in chloroplasts upon dehydration and/or ABA treatment of vegetative tissue [28].

In spite of the several bioprotection hypotheses formulated, the effectiveness mechanisms are still not fully understood. Green and Angell [29] suggest that the higher value of the glass transition temperature of trehalose and its mixtures with water, in comparison with that of the other disaccharides, is the only reason for its superior bioprotectant effectiveness: this would imply that, at a given temperature, the glass transition for trehalose mixtures always occurs at a higher water content. As a matter of fact such a hypothesis alone is not entirely satisfactory because of the many counter examples. For example dextran [30], a linear polysaccharide with $\alpha(1-6)$ glycosidic links, presents even a higher $T_{g}$ value but does not show a comparable bioprotective action.

Crowe et al. [31] formulated the hypothesis that a direct interaction between the sugars and the object of protection occurs. More specifically their "water replacement hypothesis" justifies the trehalose protective function with the existence of direct hydrogen bonding of trehalose with the polar head groups of the lipids as water does. This hypothesis was supported by some simulation studies [32], which argue that the structure of trehalose is perfectly adaptable to the tetrahedral coordination of pure water, whose structural and dynamical properties are not significantly affected by trehalose.

As a matter of fact many experimental findings [1120,33 ] have clearly shown that the structural and dynamical properties of water are perturbed by disaccharides. In particular, neutron diffraction results [12] showed for all disaccharides, and for trehalose to a large extent, a strong distortion of the peaks linked to the hydrogen bonded network in the partial radial distribution functions which can be attributed to the destroying of the tetrahedral coordination of pure water. Further neutron diffraction studies [33] combined with a Monte Carlo simulation by the EPSR code have provided high quality structural information revealing that the water structure is strongly perturbed by the presence of trehalose, with an effect on the water second hydration shell strongly resembling that produced by high pressure.

Raman spectroscopy $[11,20]$, by the analysis of the intramolecular $\mathrm{OH}$ stretching vibration band, shows that the addition of trehalose, in respect to the other disaccharides, more deeply destroys the tetrahedral intermolecular network of water, which by lowering temperature would give rise to ice. In particular the decomposition of the isotropic spectrum of the three disaccharide aqueous solutions into an "open" and a "closed" contribution shows that the integrated area of the "open" band is smaller in the trehalose aqueous solution, revealing that a more marked destructuring effect occurs in the presence of trehalose than in the presence of sucrose or maltose. Uchida et al. [34], detecting freezefractured replica images of disaccharide (trehalose, maltose, and sucrose) solutions, using a field-emission type transmission electron microscope (FE-TEM), have recently confirmed that trehalose molecules have a greater inhibitory effect of sucrose on the growth of ice crystals.

As far as dynamics is concerned, preliminary QENS results on disaccharides solutions $[12,15,20]$ indicate that also the water dynamics is strongly affected by the presence of disaccharides and in particular by trehalose. Confirmation has been furnished by molecular dynamics simulation studies performed by Bordat et al. [35]. In their work the orientational order parameter and the dynamical structure factor have been combined to differentiate the actions of trehalose, sucrose, and maltose. They showed for trehalose a higher distortion of the hydrogen bonded network of water from its tetrahedrality and that the relaxation times of water in the presence of disaccharides result in 1.2 to 10 times longer than those of pure water; in addition, water in presence of trehalose shows the longest relaxation times [35].

Cordone et al. [36] and Cottone et al. [37] by complementary techniques showed that the mean-square displacements of protein atoms are reduced by the presence of trehalose and that sugar binds to the protein through single hydrogen bonds giving rise to water molecules localized at the protein interface; in other words, sugar matrices lock the surface of the protein hindering large amplitude solvent coupled protein motions.

Caliskan et al. [38] have investigated the influence of glycerol and trehalose on lysozyme by Raman scattering, focusing particularly the attention on the low-frequency spectra. They showed that protein is coupled dynamically to trehalose and to glycerol and that glycerol provides superior suppression of protein dynamics than trehalose does at low temperature, while trehalose appears to be more effective at higher temperatures.

Cicerone et al. [39, 40] showed that adding small amounts of a low $T_{g}$ diluent to bioprotective glass leads to a reduced $T_{g}$, but an increased stability for enzymes (horseradish peroxidase and Yeast alcohol dehydrogenase, YADH) sequestered in the glass; in particular their findings indicate that it is the suppression of the local (i.e., on a short-wavelength scale $Q>0.71 \mathrm{~A}$ ), fast (i.e., high frequency $>200 \mathrm{MHz}$ ) dynamics that correlates with protein stability.

Today one of the challenges in biophysics is to uncover the precise molecular mechanisms underlying biological processes which is one of the major goals in modern biophysics. However, major roadblocks still exist on the way. Among those is the difficulty that one often encounters in characterizing complex dynamics of biological processes. In 
this framework the present review work is collocated, since the main objective is to open up new horizons for research by identifying a correlation among biomolecular dynamics, stabilization processes, and surviving strategies. More specifically we focus the attention on the use of a sophisticated physical methodology, such as the elastic neutron scattering (ENS) to investigate the dynamical properties of trehalose, compared to its homologous disaccharides, maltose and sucrose, and a class of extremophiles.

Temperature creates a series of challenges, from the structural devastation wrought by ice crystals at one extreme, to the denaturation of biomolecules at the other. High temperature can denature proteins and nucleic acids, increase the fluidity of membranes to lethal levels, while in plants chlorophyll degrades, excluding photosynthesis [41]. At low temperatures with nucleation, water freezes and the resulting ice crystals can rip cell membranes with almost invariably lethal effects [42].

Temperature also affects the structure and function of proteins. Ways that proteins have evolved to cope with high temperatures include increasing ion-pair content, decreasing the length of surface loops, forming higher-order oligomers, and decreasing flexibility at room temperature [43-45]. On the other hand, cold-temperature adaptation of protein occurs, although not always in ways that would be predicted from thermophile enzymes. At cold temperatures proteins become more rigid, implying that enhancing flexibility can restore function [46-48].

It is well known that proteins are multifunctional in the sense that their specific amino acid sequence simultaneously determines the self-organization of the linear polypeptide chain to form a unique three-dimensional structure, a specific function and its regulation, and degradation. Correspondingly, the central issue in the adaptation of proteins and enzymes to extreme conditions is the conservation of their functional state, which is characterized by a wellbalanced compromise of stability and flexibility.

Fast atomic thermal motions on the picosecond to nanosecond timescale allow proteins to achieve the stability and motions, and, therefore, the necessary rigidity and flexibility to perform their biological functions (enzymatic activity, ion pump activity ...) $[49,50]$.

Neutron scattering is particularly suited to the study of thermal molecular motions which have been shown to be correlated with the ability of a protein to undergo functional conformational changes [51-53], since neutrons of $1 \AA$ wavelength have an energy close to $1 \mathrm{kcal} / \mathrm{mol}$. Depending on the energy resolution of the spectrometer, neutron scattering can be used to observe elastic scattering, from which meansquare fluctuations in a given time scale can be calculated; quasielastic scattering, from which correlation times of diffusion motions can be calculated; inelastic scattering, arising from vibrational modes [51-53].

In this review work an overview of ENS findings on trehalose and its homologous (maltose and sucrose) water mixtures is presented as a function of temperature and concentration. The experimental findings allow to characterize both the trehalose rigidity and flexibility, which are strictly connected to its superior bioprotective effectiveness.

\section{Experimental Section}

ENS measurements have been carried out across the glass transition temperature values on trehalose, maltose, and sucrose/water $\left(\mathrm{D}_{2} \mathrm{O}\right.$ and $\left.\mathrm{H}_{2} \mathrm{O}\right)$ mixtures by using the backscattering spectrometer IN13 at the Institute Laue Langevin (Grenoble, France). The IN13 main characteristic is the relatively high energy of the incident neutrons $(16 \mathrm{meV})$ which makes it possible to span a wide range of momentum transfer $Q\left(\leq 5.5 \AA^{-1}\right)$ with a very good energy resolution $(\sim 8 \mu \mathrm{eV})$. Therefore neutron scattering experiments on IN13 provide information on the motions of the sample hydrogen in a space-time window of $1 \AA$ and 0.1 nanosecond given by its scattering vector modulus, $Q$, range and energy resolution, and allow to characterize both flexibility (obtained from the fluctuation amplitudes) and rigidity (obtained from how fluctuations vary with temperature and expressed as a mean environmental force constant) [51-53].

Ultrapure powdered trehalose, maltose and sucrose, and $\mathrm{D}_{2} \mathrm{O}$ and $\mathrm{H}_{2} \mathrm{O}$, purchased by Aldrich-Chemie, were used to prepare solutions at a weight fraction corresponding to 6 and 19 water molecules for each disaccharide molecule.

\section{Theoretical Background}

Hydrogen atoms in a system move in a potential $V(\mathbf{r})$, where $\mathbf{r}(t)$ denotes the position vector of the particle at time $t[51$, $54,55]$.

In incoherent neutron scattering experiments the measured function is the incoherent dynamic structure factor $S_{\text {inc }}(Q, \omega)$, that is, Fourier transform of the incoherent intermediate scattering function $I(Q, t)[51,54,55]$ :

$$
I(Q, t)=\sum_{\alpha=1}^{N} x_{\alpha}\left\langle\exp \left[i Q \cdot r_{\alpha}(t)\right] \exp \left[-i Q \cdot r_{\alpha}(0)\right]\right\rangle,
$$

where $x_{\alpha} \alpha\left(\sum_{\alpha=1}^{N} x_{\alpha}=1\right)$ is the fraction of dynamically equivalent particles in the potential $V_{\alpha}(\mathbf{r})$, brackets denote the ensemble average over many trajectories for particles initially at thermal equilibrium and $r_{\alpha}(t)$ the position vector of the particle $\alpha$ at time $t$.

For small $Q$ values, in the Gaussian scattering approximation, the orientational average can be performed to eliminate angular coordinates yielding

$$
I(Q, t) \cong \sum_{\alpha=1}^{N} x_{\alpha} \exp \left[-\frac{Q^{2}}{6}\left\langle\left[r_{\alpha}(t)-r_{\alpha}(0)\right]^{2}\right\rangle\right] .
$$

Due to the polydispersity of the system, the intensity is a combination of several Gaussian functions:

$$
I(Q, t) \cong \sum_{\alpha=1}^{N} x_{\alpha} \exp \left[-\frac{Q^{2}}{3}\left\langle r_{\alpha}^{2}\right\rangle\left[1-C_{\alpha}(t)\right]\right]
$$

where $\left\langle r_{\alpha}^{2}\right\rangle$ is the equilibrium mean-square displacement and $C_{\alpha}(t)$ stationary position relaxation function. The meansquare displacement reduces to the equilibrium value for times long enough such that $C_{\alpha}(t) \rightarrow 0[51,54,55]$. 
The mean-square displacement, $\left\langle u^{2}\right\rangle$, which takes into account fluctuations of all particles in the investigated system, is given by

$$
\begin{aligned}
\left\langle u^{2}\right\rangle & =-\left.3 \frac{d\left\{\ln \left[S_{\text {inc }}^{\mathrm{el}}(Q)\right]\right\}}{d Q^{2}}\right|_{Q=0} \\
& =\sum_{\alpha=1}^{N} x_{\alpha}\left\langle r_{\alpha}^{2}\right\rangle\left[1-C_{\alpha}(\tau)\right] .
\end{aligned}
$$

Nonetheless, for a given experiment $C_{\alpha}(\tau)$ is a constant that rescales the observed mean-square displacement, and hence $C_{\alpha}(\tau)=0$ can be assumed. For simplicity in the present analysis the assumption that all particles are dynamically equivalent will be made, therefore in the previous equations $x_{\alpha}$ has been assumed equal to $1[51,54,55]$.

The mean-square displacement of a set of quantized harmonic oscillators for $T<\left(h\langle\nu\rangle /\left(2 K_{B}\right)\right)\left(h\langle\nu\rangle / K_{B}\right)$ being the Debye temperature, $K_{B}$ being the Boltzmann constant, $\langle\nu\rangle$ being the average frequency of a set of oscillators considered as an Einstein solid) is almost a constant equal to the zero-point fluctuations $h\langle v\rangle / 2 K_{\text {force }}$ (where $K_{\text {force }}$ is the average force field constant of a set of oscillators considered as an Einstein solid), whereas it linearly increases with the temperature for $T>h\langle\nu\rangle / 2 K_{B}[51,54,55]$.

In the $Q$ range in which the Gaussian model is valid, $\left\langle\Delta u^{2}\right\rangle Q^{2}<2$, where $\left\langle\Delta u^{2}\right\rangle=\left\langle u^{2}(T)\right\rangle-\left\langle u^{2}(20 K)\right\rangle$ is the total mean-square displacement of hydrogens, and $\left.S_{\text {inc }}^{\text {el }}(Q) \propto \exp L-\left\langle\Delta u^{2} / 6\right\rangle Q^{2}\right\rfloor$, the mean-square displacement behavior can be fitted within the framework of the harmonic approximation:

$$
\left\langle\Delta u^{2}(T)\right\rangle=\left[\frac{h\langle v\rangle}{2 K_{\text {force }}}\right] \operatorname{coth}\left(\frac{h\langle\nu\rangle}{2 K_{B} T}-1\right) .
$$

Because a force constant is not defined for anharmonic motions, an operational approach in which the "resilience" of an anharmonic environment is quantified by a pseudoforce constant $\langle k\rangle$ calculated from the derivative of the scan at $T$ according to $\langle k\rangle=0.00138 /\left(d\left\langle u^{2}\right\rangle / d T\right)$ has been suggested $[51,54,55]$.

As the deviation from Gaussian behavior increases with temperature, the motion involves jumping of hydrogens to distinct sites of different energy, the expression of the integrated intensity as a function of $Q$ being $[51,54,55]$ :

$$
\begin{aligned}
I_{\mathrm{el}}(Q) & =S(Q, \omega=0) \\
& =A \exp \left(-Q^{2}\left\langle u^{2}\right\rangle\right)\left[1-2 p_{1} p_{2}\left(\frac{\sin (Q d)}{Q d}\right)\right],
\end{aligned}
$$

where $p_{1}$ and $p_{2}$ are the probabilities to find the scattering particles on the ground state and the excited state, respectively, and $d$ is the distance between the two potential minima.

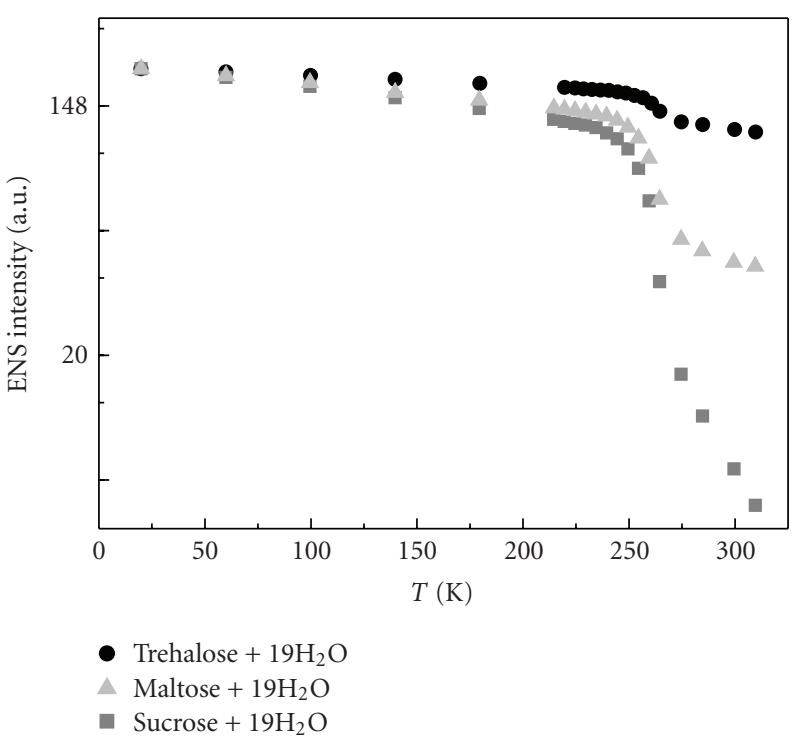

(a)

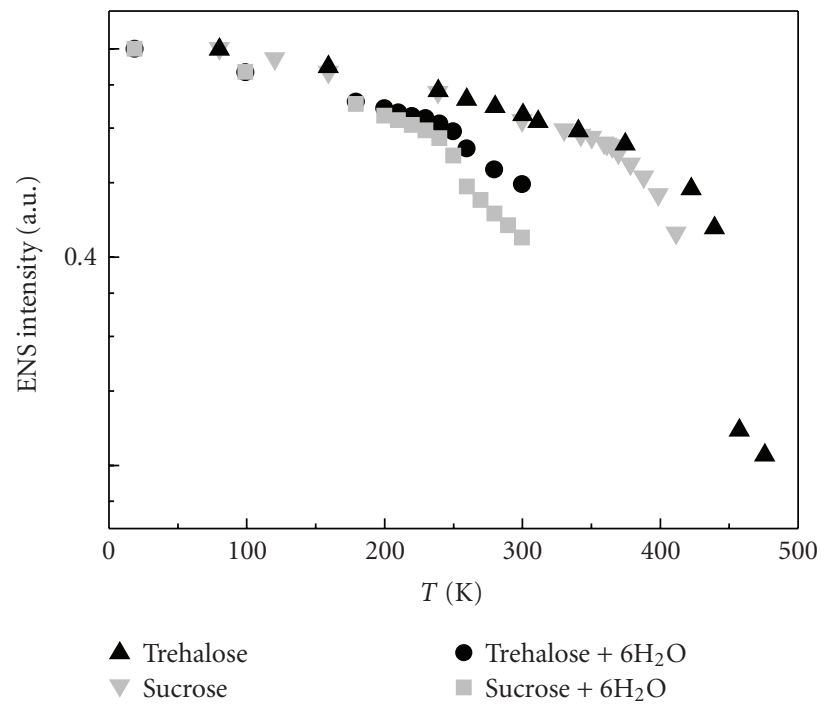

(b)

FIGURE 1: ENS spectra of (a) trehalose, maltose, and sucrose $/ 19 \mathrm{H}_{2} \mathrm{O}$ mixtures and (b) trehalose and sucrose, trehalose and sucrose $/ 6 \mathrm{H}_{2} \mathrm{O}$ mixtures as a function of temperature.

\section{Results and Discussion}

Figure 1 shows the ENS intensity versus temperature and concentration for trehalose, maltose, and sucrose $/ \mathrm{H}_{2} \mathrm{O}$ mixtures, while in Figure 2 the ENS intensity surfaces of trehalose, maltose, and sucrose $/ 19 \mathrm{H}_{2} \mathrm{O}$ mixtures as a function of temperature and exchanged wavevector $Q$ are shown.

It is evident that a dynamical transition occurs for the three investigated systems; for the trehalose mixture at $T \sim 238 \mathrm{~K}$, whereas for maltose and sucrose mixtures at $T \sim 235 \mathrm{~K}$ and $T \sim 233 \mathrm{~K}$, respectively. Below the onset temperature the elastic intensity has the Gaussian form expected for a harmonic solid. The decrease in the elastic intensity above the dynamical transition temperature can 


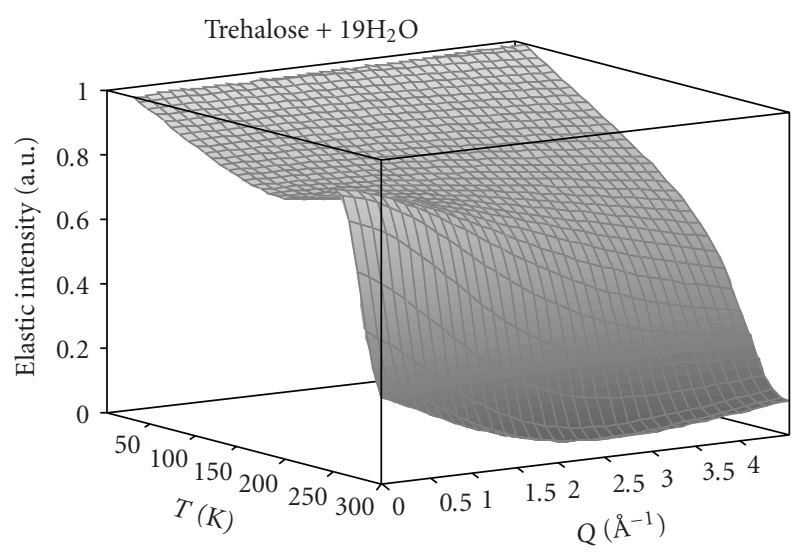

(a)

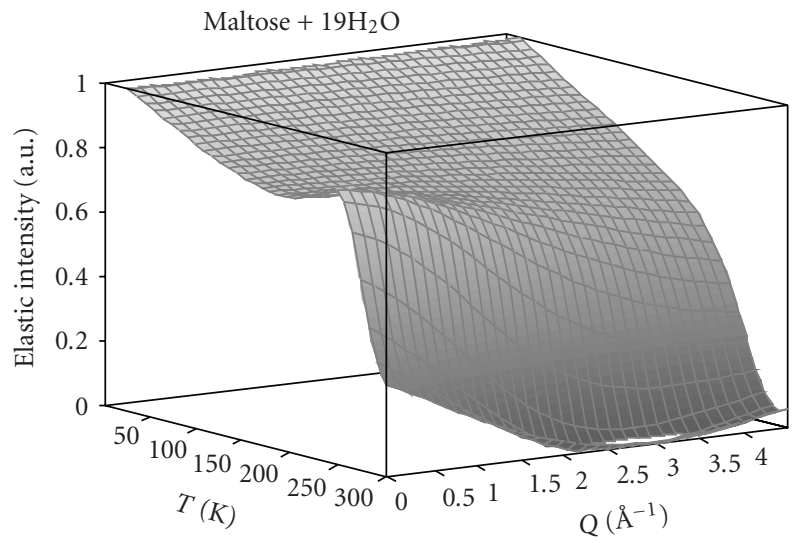

(b)

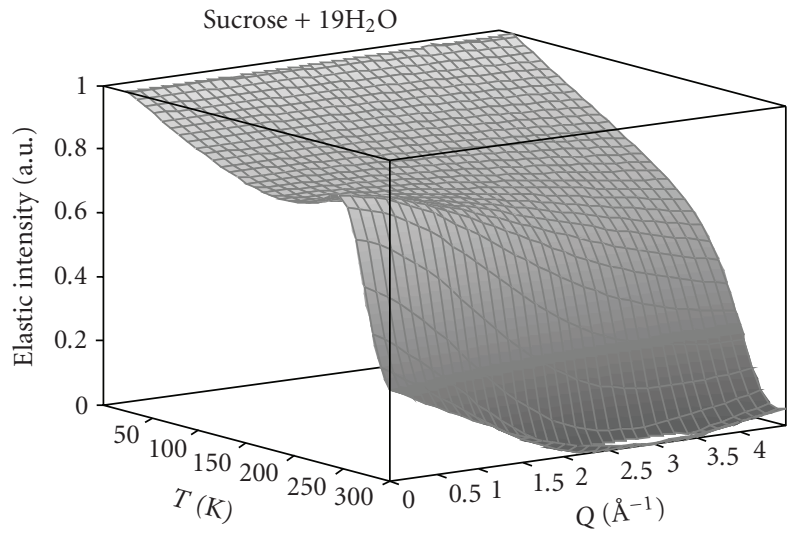

(c)

FIGURE 2: ENS 3D-spectra of trehalose, maltose, and sucrose $/ 19 \mathrm{H}_{2} \mathrm{O}$ mixtures as a function of temperature and Q.

be attributed to the excitation of new degrees of freedom, especially at low $Q$ and, as suggested by Figures 1 and 2, is very less marked in the case of trehalose/water mixture than for the other disaccharide/water mixtures for all the investigated concentration values. This circumstance clearly indicates that trehalose shows a larger structural resistance to temperature changes as it occurs in "stronger" systems and presents a higher "rigidity" in comparison with the

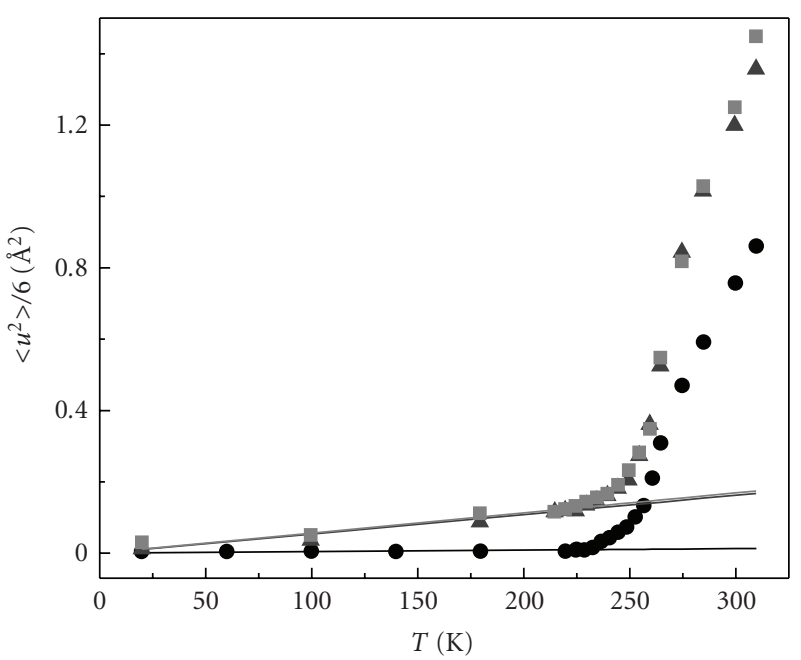

- Trehalose $+19 \mathrm{H}_{2} \mathrm{O}$

- Maltose $+19 \mathrm{H}_{2} \mathrm{O}$

- Sucrose $+19 \mathrm{H}_{2} \mathrm{O}$

(a)

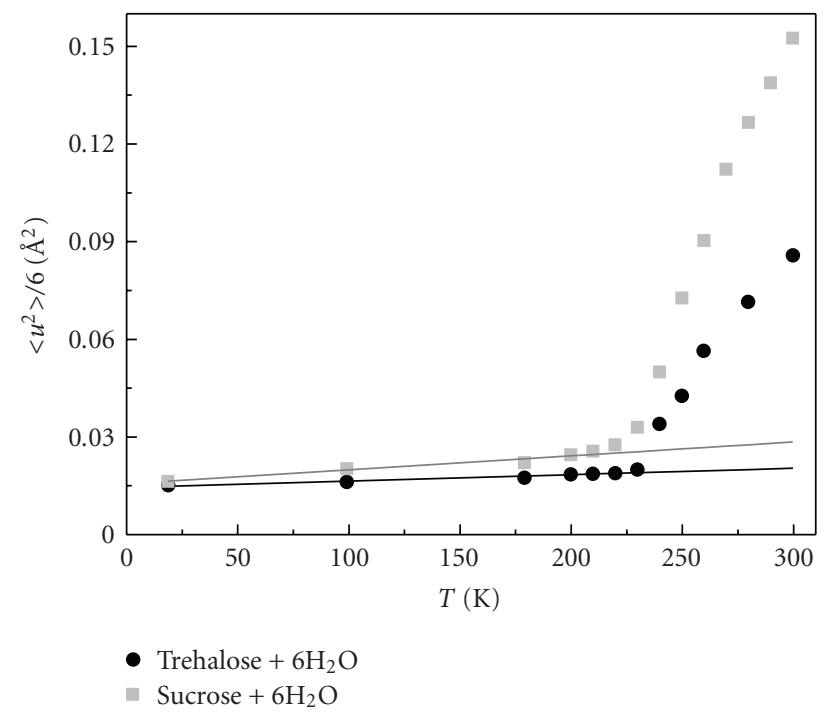

(b)

FIGURE 3: Temperature dependence of the derived mean-square displacements for (a) trehalose, maltose, and sucrose $/ 19 \mathrm{H}_{2} \mathrm{O}$ mixtures and (b) trehalose and sucrose $/ 6 \mathrm{H}_{2} \mathrm{O}$ mixtures.

other disaccharides, namely, the maltose $/ \mathrm{H}_{2} \mathrm{O}$ mixture and the sucrose $/ \mathrm{H}_{2} \mathrm{O}$ mixture, this latter showing the "softest" character.

Figure 3 shows the temperature dependence of the derived mean-square displacements for trehalose, maltose, and sucrose $/ \mathrm{H}_{2} \mathrm{O}$ mixtures at different concentration values.

By fitting the ENS data shown in Figure 3 according to (6), we obtain the values of $K=0.40 \mathrm{~N} / \mathrm{m}, K=0.25 \mathrm{~N} / \mathrm{m}$, and $K=0.22 \mathrm{~N} / \mathrm{m}$ for the average force field constant for trehalose, maltose, and sucrose mixtures, respectively, indicating the trehalose $/ \mathrm{H}_{2} \mathrm{O}$ system as the strongest system. 
In the following we shall connect the bioprotectant effectiveness of disaccharide $/ \mathrm{H}_{2} \mathrm{O}$ mixtures to the "fragility degree" of these systems. For this purpose we use an operative definition for fragility, based on the evaluation by neutron scattering of the temperature dependence of the mean-square displacements. The definition, differently from many other phenomenological parameters as, for example, resilience introduced in literature, allows to connect a transport quantity, that is, viscosity, with an atomic quantity, the nanoscopic mean-square displacement.

It is well known that the behavioral properties of a glass-forming system are often described by the $(3 N+1)$ dimensional potential energy hypersurface in the configurational space $[18,56,57]$. The complexity of the energy landscape, explored by the system, is correlated with the density of the minima of the hypersurface (degeneracy $\left.\propto \Delta C_{p}\left(T_{g}\right)\right)$ and with the distribution of the barrier heights between them $\Delta \mu$. These features determine the structural sensitivity of a system to temperature changes in approaching the glass transition, namely, its fragility. The latter is operatively defined as

$$
m=\left.\frac{d \log \eta}{d\left(T_{g} / T\right)}\right|_{T=T_{g}^{+}}=\frac{\Delta C_{p}\left(T_{g}\right)}{\Delta \mu} .
$$

In this frame Angell's classification of glass-forming systems takes particular relevance $[56,57]$. In such a classification, based on the choice of an invariant viscosity at the scaling temperature $T_{g}\left(\eta\left(T_{g}\right)=10^{13}\right.$ poise), the departure from the Arrhenius law is taken as a signature of the degree of fragility of the system [56, 57]. An Arrhenius behavior of viscosity in the $T_{g}$-scaled plot and a small heat capacity variation $\Delta C_{p}\left(T_{g}\right)$ characterize the strongest systems, whereas a large departure from Arrhenius law and a large heat capacity variation $\Delta C_{p}\left(T_{g}\right)$ characterize the most fragile ones. Between these two limiting cases, intermediate behaviors are interpreted in terms of different kinetic and thermodynamic contributions: thermodynamically strong (fragile) and kinetically fragile (strong) systems are characterized by a low (high) configurational degeneracy and small (large) barrier heights.

Now starting from the works on Selenium $[58,59]$, a clear correlation between viscosity and the atomic meansquare displacement is found. The following picture for the elementary flow process (the $\alpha$-relaxation) is suggested: a given atom is jumping back and forth in the fast processes ( $\beta$-relaxation motions). Its probability distribution in that fast motion is a Gaussian with the mean-square amplitude $\left\langle u^{2}\right\rangle_{\text {loc }}$, defined as the difference between the mean-square displacement of the ordered and disordered phase [60]:

$$
\left\langle u^{2}\right\rangle_{\text {loc }}=\left\langle u^{2}\right\rangle_{\text {anharm }}-\left\langle u^{2}\right\rangle_{\text {harm }} .
$$

If the amplitude of that fast motion exceeds by chance a critical displacement $u_{0}$, a local structural reconfiguration (the $\alpha$-relaxation) takes place. Assuming the time scale of the fast motion to be independent on temperature, the waiting time for the occurrence of an $\alpha$-process at a given atom is proportional to the probability to find the atom outside of

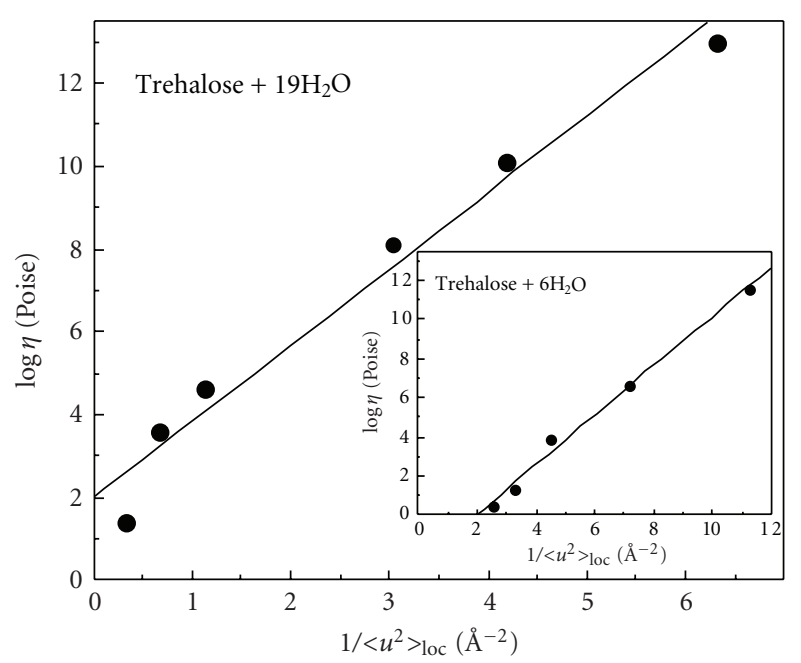

FIGURE 4: Linear dependence of the logarithm of the viscosity on $1 /\left\langle u^{2}\right\rangle_{\text {loc }}$ for trehalose $/ 19 \mathrm{H}_{2} \mathrm{O}$ mixture. In the insert the logarithm of the viscosity as a function of $1 /\left\langle u^{2}\right\rangle_{\text {loc }}$ for trehalose $/ 6 \mathrm{H}_{2} \mathrm{O}$ mixture is shown. The solid lines indicate the best fit of the experimental data.

the sphere with radius $u_{0}$. In this picture, one obtains the viscosity [60]

$$
\eta=\eta_{0} \exp \left\lfloor\frac{u_{0}^{2}}{\left\langle u^{2}\right\rangle_{\mathrm{loc}}}\right\rfloor .
$$

The result obtained for trehalose $/ \mathrm{H}_{2} \mathrm{O}$ mixtures at different concentration values is shown in Figure 4.

Taking into account (7)-(9), the "fragility" degree by elastic neutron scattering is operatively defined as

$$
M=\left.\frac{d\left(u_{0}^{2} /\left\langle u^{2}\right\rangle_{\mathrm{loc}}\right)}{d\left(T_{g} / T\right)}\right|_{T=T_{g}^{+}} .
$$

Obviously such a definition would imply a fragility parameter depending on the instrumental resolution, but on the other hand we are interested on a comparison ceteris paribus of the fragility degree and such a comparison is meaningful both when an identical experimental set-up for IN13 is employed. As we will see the experimental set-up of IN13 with an elastic energy resolution of $8 \mu \mathrm{eV}$ (FWHM) furnishes values in agreement with those evaluated by viscosity measurements in a limited temperature range.

From (10) we evaluate a fragility parameter $M$ of 302 for the trehalose $+19 \mathrm{H}_{2} \mathrm{O}$ mixture, of 346 for the maltose + $19 \mathrm{H}_{2} \mathrm{O}$ mixture, and of 355 for the sucrose $+19 \mathrm{H}_{2} \mathrm{O}$ mixture. Employing less wide viscosity data sets, for trehalose $/ \mathrm{D}_{2} \mathrm{O}$ and sucrose $/ \mathrm{D}_{2} \mathrm{O}$ mixtures at a concentration value corresponding to 19 water molecules for each disaccharide molecule, we obtain a fragility parameter $M$ of 272 and of 295 , respectively, whereas for the trehalose $/ \mathrm{H}_{2} \mathrm{O}$ and sucrose $/ \mathrm{H}_{2} \mathrm{O}$ mixtures at a concentration value corresponding to 6 water molecules for each disaccharide molecule we obtain for the fragility parameter $M$ the value of 241 and of 244 , respectively. 


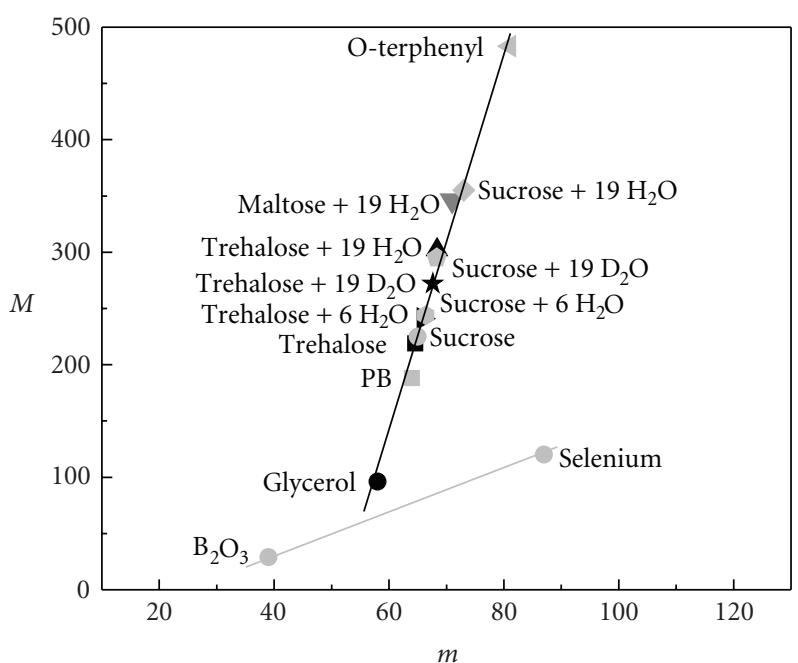

FIGURE 5: Fragility parameter $M$, as obtained by ENS experiments, versus the fragility parameter $m$, as obtained by viscosity measurements. The glycerol, PB, disaccharides, and OTP are experimental data obtained by using the IN13 spectrometer at an instrumental elastic energy resolution of $8 \mu \mathrm{eV}$; the $\mathrm{B}_{2} \mathrm{O}_{3}$ and Selenium data are experimental data obtained by using the IN6 spectrometer at a instrumental elastic energy resolution of $150-200 \mu \mathrm{eV}$. The solid black and grey lines indicate the best fits.

Figure 5 shows the obtained $M$ values as a function of $m$. The obtained values indicate that the present operative definition for fragility furnishes an excellent direct proportionality between $M$ and $m$.

\section{Conclusions}

Life under extreme conditions is limited primarily by the stability of macromolecules and their ability to exert their biochemical functions under nonconventional conditions that normally lead to the collapse of structures or to functional inactivation. Stability refers to the maintenance of the spatially defined functional state under extreme conditions. The central issue in the adapatation of biomolecules to extreme conditions is the conservation of their functional state, which means a well-balanced compromise between stability and flexibility.

Maintaining the appropriate balance between molecular stability and structural flexibility is necessary for protein functions. Stability is needed to ensure the appropriate geometry for ligand binding, as well as to avoid denaturation, while flexibility is necessary to allow a catalysis at a metabolically appropriate rate. However, the relationship between activity, flexibility, and stability are still the subject of controversy. A definition of flexibility in terms of type, amplitude, and time scale of molecular motions related to the catalytic efficiency is therefore helpful in understanding these relationships.

The dynamic nature of hyperthermostable proteins may be the key to unraveling the mechanism responsible for the delicate balance between rigidity, which is related to heat resistance, and molecular fluctuations at high temperatures, which account for biological function.
Tehei et al. [61-64] have used ENS to characterize the mean motions of the entire cellular macromolecular population in vivo. They have studied the adaptation mechanism effects on the macromolecular dynamics on whole live bacteria: the psychrophile Aquaspirillum arcticum, the mesophiles Escherichia coli and Proteus mirabilis, the thermophile Thermus thermophilus, and the hyperthermophile Aquifex pyrofilus.

The mean effective force constant values obtained for these extremophiles are $\left\langle k^{\prime}\right\rangle=0.21 \pm 0.03 \mathrm{~N} / \mathrm{m}$ for $A$. arcticum, $0.42 \pm 0.01 \mathrm{~N} / \mathrm{m}$ for $E$. coli, $0.39 \pm 0.01 \mathrm{~N} / \mathrm{m}$ for P. mirabilis, $0.67 \pm 0.011 \mathrm{~N} / \mathrm{m}$ for $T$. thermophilus, and $0.60 \pm 0.01 \mathrm{~N} / \mathrm{m}$ for $A$. pyrofilus cells [51]. Tehei et al. [61-64] have therefore shown, in vivo, the existence of a strong correlation between resilience and adaptation to a physiological temperature.

Tehei et al. have concluded that psychrophile proteins have adapted to achieve increased structural flexibility, necessary for activity at low temperature, and that the enhanced thermal stability of thermophile proteins is associated with increased rigidity. Thermophile enzymes are also characterized by a higher temperature of maximum activity. On the other hand the more rigid hyperthermophilic enzyme would then require higher temperatures in order to achieve the requisite conformational flexibility for activity [61-64].

The present review paper is based on the overview of the remarkable quantities which ENS allows to determine in order to characterize the dynamical properties, the flexibility, and fragility of biomolecules, that is, bioprotectant systems.

The observed dynamical transition marks a cross-over in molecular fluctuations between Gaussian and non-Gaussian behavior of the ENS disaccharide profiles. These findings furnish useful information on the different nature of the involved dynamical processes in bioprotection that can justify the higher trehalose "cryptobiotic" effectiveness. In fact the best cryptoprotectant effectiveness of trehalose is ascribed to the lower flexibility and fragile character of the matrix in which biostructures are immersed, that is, of the trehalose/water mixture. This circumstance implies a better attitude to encapsulate biostructures in more rigid and more temperature insensitive structures in respect to maltose and sucrose/water mixtures.

\section{Acknowledgments}

With the financial support of the Minister of Foreign Affairs, General Direction for the Promotion and Cultural Cooperation (Italy-South Africa Project "Physical and biochemical studies of molecular mechanisms of bioprotection in drought-resistant plants"). This work has been realized in the framework of the MIUR-International University System art. 23 n. 262 Project titled "Study of the stabilisation and conservation effectiveness of trehalose on high added value products". F. Migliardo gratefully acknowledges UNESCOL'Oréal for the International Fellowship for Young Women in Life Sciences 2008. The authors gratefully acknowledge the Institut Laue-Langevin ILL (Grenoble, France) for dedicated runs at IN13 spectrometer. 


\section{References}

[1] S. L. Miller and A. Lazcano, "The origin of life-did it occur at high temperatures?" Journal of Molecular Evolution, vol. 41, no. 6, pp. 689-692, 1995.

[2] P. Forterre, "A hot topic: the origin of hyperthermophiles," Cell, vol. 85, no. 6, pp. 789-792, 1996.

[3] T. D. Brock, K. M. Brock, R. T. Belly, and R. L. Weiss, "Sulfolobus: a new genus of sulfur-oxidizing bacteria living at low $\mathrm{pH}$ and high temperature," Archiv für Mikrobiologie, vol. 84, no. 1, pp. 54-68, 1972.

[4] K. O. Stetter, "History of discovery of the first hyperthermophiles," Extremophiles, vol. 10, no. 5, pp. 357-362, 2006.

[5] K. Kashefi and D. R. Lovley, "Extending the upper temperature limit for life," Science, vol. 301, no. 5635, p. 934, 2003.

[6] M. A. Arnott, R. A. Michael, C. R. Thompson, D. W. Hough, and M. J. Danson, "Thermostability and thermoactivity of citrate synthases from the thermophilic and hyperthermophilic archaea, thermoplasma acidophilum and Pyrococcus furiosus," Journal of Molecular Biology, vol. 304, no. 4, pp. 657-668, 2000.

[7] D. Madern, C. Ebel, and G. Zaccai, "Halophilic adaptation of enzymes," Extremophiles, vol. 4, no. 2, pp. 91-98, 2000.

[8] D. W. Grogan, "Hyperthermophiles and the problem of DNA instability," Molecular Microbiology, vol. 28, no. 6, pp. 10431049, 1998.

[9] R. M. Daniel and D. A. Cowan, "Biomolecular stability and life at high temperatures," Cellular and Molecular Life Sciences, vol. 57, no. 2, pp. 250-264, 2000.

[10] C. Vieille and G. J. Zeikus, "Hyperthermophilic enzymes: sources, uses, and molecular mechanisms for thermostability," Microbiology and Molecular Biology Reviews, vol. 65, no. 1, pp. $1-43,2001$.

[11] C. Branca, A. Faraone, S. Magazù, et al., "Structural and dynamical properties of trehalose-water solutions: anomalous behaviour and molecular models ," Recent Research Developments in Physical Chemistry, vol. 3, pp. 361-403, 1999.

[12] C. Branca, S. Magazù, and F. Migliardo, "New perspectives on bioprotectant complex molecules: spectroscopic findings," Recent Research Developments in Physical Chemistry, vol. 6, pp. 35-73, 2002.

[13] S. Magazù, F. Migliardo, and A. J. Ramirez-Cuesta, "Inelastic neutron scattering study on bioprotectant systems," Journal of the Royal Society Interface, vol. 2, no. 5, pp. 527-532, 2005.

[14] A. Lerbret, P. Bordat, F. Affouard, M. Descamps, and F. Migliardo, "How homogeneous are the trehalose, maltose, and sucrose water solutions? An insight from molecular dynamics simulations," The Journal of Physical Chemistry B, vol. 109, no. 21, pp. 11046-11057, 2005.

[15] S. Magazù, F. Migliardo, and M. T. F. Telling, “ $\alpha, \alpha$-trehalosewater solutions. VIII. Study of the diffusive dynamics of water by high-resolution quasi elastic neutron scattering," The Journal of Physical Chemistry B, vol. 110, no. 2, pp. 1020-1025, 2006.

[16] S. Magazù, F. Migliardo, and A. J. Ramirez-Cuesta, "Changes in vibrational modes of water and bioprotectants in solution," Biophysical Chemistry, vol. 125, no. 1, pp. 138-142, 2007.

[17] C. Branca, S. Magazù, G. Maisano, F. Migliardo, P. Migliardo, and G. Romeo, " $\alpha, \alpha$-trehalose/water solutions. 5. Hydration and viscosity in dilute and semidilute disaccharide solutions," The Journal of Physical Chemistry B, vol. 105, no. 41, pp. 10140-10145, 2001.

[18] S. Magazù, G. Maisano, F. Migliardo, and C. Mondelli, "Meansquare displacement relationship in bioprotectant systems by elastic neutron scattering," Biophysical Journal, vol. 86, no. 5, pp. 3241-3249, 2004.

[19] B. Varga, F. Migliardo, E. Takacs, B. Vertessy, S. Magazù, and C. Mondelli, "Neutron scattering studies on dUTPase complex in the presence of bioprotectant systems," Chemical Physics, vol. 345, no. 2-3, pp. 250-258, 2008.

[20] S. Magazù, F. Migliardo, and M. T. F. Telling, "Structural and dynamical properties of water in sugar mixtures," Food Chemistry, vol. 106, no. 4, pp. 1460-1466, 2008.

[21] A. G. Hirsh, "Vitrification in plants as a natural form of cryoprotection," Cryobiology, vol. 24, no. 3, pp. 214-228, 1987.

[22] J. H. Crowe, L. M. Crowe, and D. Chapman, "Preservation of membranes in anhydrobiotic organisms: the role of trehalose," Science, vol. 223, no. 4637, pp. 701-703, 1984.

[23] K. B. Storey and J. M. Storey, "Natural freeze tolerance in ectothermic vertebrates," Annual Review of Physiology, vol. 54, pp. 619-637, 1992.

[24] D. A. Wharton, Life at the Limits: Organisms in Extreme Environments, Cambridge University Press, Cambridge, UK, 2002.

[25] W. R. Miller, "Tardigrades: bears of the moss," The Kansas School Naturalist, vol. 43, no. 3, pp. 1-15, 1997.

[26] B. Leyman, P. Van Dijck, and J. M. Thevelein, "An unexpected plethora of trehalose biosynthesis genes in Arabidopsis thaliana," Trends in Plant Science, vol. 6, no. 11, pp. 510-513, 2001.

[27] R. Zentella, J. O. Mascorro-Gallardo, P. Van Dijck, et al., "A Selaginella lepidophylla trehalose-6-phosphate synthase complements growth and stress-tolerance defects in a yeast tps1 mutant," Plant Physiology, vol. 119, no. 4, pp. 1473-1482, 1999.

[28] M. Kasuga, Q. Liu, S. Miura, K. Yamaguchi-Shinozaki, and K. Shinozaki, "Improving plant drought, salt, and freezing tolerance by gene transfer of a single stress-inducible transcription factor," Nature Biotechnology, vol. 17, no. 3, pp. 287-291, 1999.

[29] J. L. Green and C. A. Angell, "Phase relations and vitrification in saccharide-water solutions and the trehalose anomaly," The Journal of Physical Chemistry, vol. 93, no. 8, pp. 2880-2882, 1989.

[30] J. H. Crowe, F. A. Hoekstra, K. H. N. Nguyen, and L. M. Crowe, "Is vitrification involved in depression of the phase transition temperature in dry phospholipids?" Biochimica et Biophysica Acta, vol. 1280, no. 2, pp. 187-196, 1996.

[31] L. M. Crowe, D. S. Reid, and J. H. Crowe, "Is trehalose special for preserving dry biomaterials?” Biophysical Journal, vol. 71, no. 4, pp. 2087-2093, 1996.

[32] M. C. Donnamaria, E. I. Howard, and J. R. Grigera, "Interaction of water with $\alpha, \alpha$-trehalose in solution: molecular dynamics simulation approach," Journal of the Chemical Society, Faraday Transactions, vol. 90, no. 18, pp. 2731-2735, 1994.

[33] S. E. Pagnotta, M. A. Ricci, F. Bruni, S. McLain, and S. Magazù, "Water structure around trehalose," Chemical Physics, vol. 345, no. 2-3, pp. 159-163, 2008.

[34] T. Uchida, M. Nagayama, T. Shibayama, and K. Gohara, "Morphological investigations of disaccharide molecules for growth inhibition of ice crystals," Journal of Crystal Growth, vol. 299, no. 1, pp. 125-135, 2007.

[35] P. Bordat, A. Lerbret, J.-P. Demaret, F. Affouard, and M. Descamps, "Comparative study of trehalose, sucrose and maltose in water solutions by molecular modelling," Europhysics Letters, vol. 65, no. 1, pp. 41-47, 2004. 
[36] L. Cordone, G. Cottone, S. Giuffrida, G. Palazzo, G. Venturoli, and C. Viappiani, "Internal dynamics and protein-matrix coupling in trehalose-coated proteins," Biochimica et Biophysica Acta, vol. 1749, no. 2, pp. 252-281, 2005.

[37] G. Cottone, G. Ciccotti, and L. Cordone, "Protein-trehalosewater structures in trehalose coated carboxy-myoglobin," Journal of Chemical Physics, vol. 117, no. 21, pp. 9862-9866, 2002.

[38] G. Caliskan, D. Mechtani, J. H. Roh, et al., "Protein and solvent dynamics: how strongly are they coupled?" Journal of Chemical Physics, vol. 121, no. 4, pp. 1978-1983, 2004.

[39] M. T. Cicerone, A. Tellington, L. Trost, and A. Sokolov, "Substantially improved stability of biological agents in dried form," BioProcess International, vol. 1, pp. 36-47, 2003.

[40] M. T. Cicerone and C. L. Soles, "Fast dynamics and stabilization of proteins: binary glasses of trehalose and glycerol," Biophysical Journal, vol. 86, no. 6, pp. 3836-3845, 2004.

[41] L. J. Rothschild and R. L. Mancinelli, "Life in extreme environments," Nature, vol. 409, no. 6823, pp. 1092-1101, 2001.

[42] S. Koutsopoulos, J. van der Oost, and W. Norde, "Temperature-dependent structural and functional features of a hyperthermostable enzyme using elastic neutron scattering," Proteins: Structure, Function and Bioinformatics, vol. 61, no. 2, pp. 377-384, 2005.

[43] L. D. Unsworth, J. van der Oost, and S. Koutsopoulos, "Hyperthermophilic enzymes—stability, activity and implementation strategies for high temperature applications," The FEBS Journal, vol. 274, no. 16, pp. 4044-4056, 2007.

[44] J. M. Bragger, R. M. Daniel, T. Coolbear, and H. W. Morgan, "Very stable enzymes from extremely thermophilic archaebacteria and eubacteria," Applied Microbiology and Biotechnology, vol. 31, no. 5-6, pp. 556-561, 1989.

[45] H. R. Badr, K. A. Sims, and M. W. W. Adams, "Purification and characterization of sucrose $\alpha$-glucohydrolase (invertase) from the hyperthermophilic archaeon Pyrococcus furiosus," Systematic and Applied Microbiology, vol. 17, no. 1, pp. 1-6, 1994.

[46] D. Tronelli, E. Maugini, F. Bossa, and S. Pascarella, "Structural adaptation to low temperatures-analysis of the subunit interface of oligomeric psychrophilic enzymes," The FEBS Journal, vol. 274, no. 17, pp. 4595-4608, 2007.

[47] A. Szilágyi and P. Závodszky, "Structural differences between mesophilic, moderately thermophilic and extremely thermophilic protein subunits: results of a comprehensive survey," Structure, vol. 8, no. 5, pp. 493-504, 2000.

[48] G. Feller, D. D'Amico, and C. Gerday, “Thermodynamic stability of a cold-active $\alpha$-amylase from the antarctic bacterium Alteromonas haloplanctis," Biochemistry, vol. 38, no. 14, pp. 4613-4619, 1999.

[49] C. L. Brooks, M. Karplus, and B. M. Pettitt, Eds., Proteins: A Theoretical Perspective of Dynamics, Structure and Function, vol. 71 of Advances in Chemical Physics, John Wiley \& Sons, New York, NY, USA, 1988.

[50] U. Lehnert, V. Réat, M. Weik, G. Zaccaï, and C. Pfister, "Thermal motions in bacteriorhodopsin at different hydration levels studied by neutron scattering: correlation with kinetics and light-induced conformational changes," Biophysical Journal, vol. 75, no. 4, pp. 1945-1952, 1998.

[51] G. Zaccai, "How soft is a protein? A protein dynamics force constant measured by neutron scattering," Science, vol. 288, no. 5471, pp. 1604-1607, 2000.
[52] W. Doster, S. Cusack, and W. Petry, "Dynamical transition of myoglobin revealed by inelastic neutron scattering," Nature, vol. 337, no. 6209, pp. 754-756, 1989.

[53] J. C. Smith, "Protein dynamics: comparison of simulations with inelastic neutron scattering experiments," Quarterly Reviews of Biophysics, vol. 24, no. 3, pp. 227-291, 1991.

[54] D. J. Bicout and G. Zaccai, "Protein flexibility from the dynamical transition: a force constant analysis," Biophysical Journal, vol. 80, no. 3, pp. 1115-1123, 2001.

[55] V. Réat, H. Patzelt, M. Ferrand, C. Pfister, D. Oesterhelt, and G. Zaccai, "Dynamics of different functional parts of bacteriorhodopsin: $\mathrm{H}_{-}^{2} \mathrm{H}$ labeling and neutron scattering," Proceedings of the National Academy of Sciences of the United States of America, vol. 95, no. 9, pp. 4970-4975, 1998.

[56] C. A. Angell, P. H. Poole, and J. Shao, "Glass-forming liquids, anomalous liquids, and polyamorphism in liquids and biopolymers," Il Nuovo Cimento D, vol. 16, no. 8, pp. 9931025, 1994.

[57] C. A. Angell, "Glassforming liquids with microscopic to macroscopic two-state complexity," Progress of Theoretical Physics, vol. 126, pp. 1-8, 1997.

[58] G. Galli, P. Migliardo, R. Bellissent, and W. Reichardt, "DebyeWaller factor through the glass transition temperature in aselenium, by incoherent inelastic neutron scattering," Solid State Communications, vol. 57, no. 3, pp. 195-198, 1986.

[59] M. Federico, G. Galli, S. Magazù, D. Majolino, and E. Burattini, "Study of the glass transition region in amorphous selenium by EXAFS," Il Nuovo Cimento D, vol. 10, no. 4, pp. 425-434, 1988.

[60] U. Buchenau and R. Zorn, "A relation between fast and slow motions in glassy and liquid selenium," Europhysics Letters, vol. 18, no. 6, pp. 523-528, 1992.

[61] M. Tehei and G. Zaccai, "Adaptation to high temperatures through macromolecular dynamics by neutron scattering," The FEBS Journal, vol. 274, no. 16, pp. 4034-4043, 2007.

[62] M. Tehei, D. Madern, B. Franzetti, and G. Zaccai, "Neutron scattering reveals the dynamic basis of protein adaptation to extreme temperature," Journal of Biological Chemistry, vol. 280, no. 49, pp. 40974-40979, 2005.

[63] M. Tehei, D. Madern, C. Pfister, and G. Zaccai, "Fast dynamics of halophilic malate dehydrogenase and BSA measured by neutron scattering under various solvent conditions influencing protein stability," Proceedings of the National Academy of Sciences of the United States of America, vol. 98, no. 25, pp. 14356-14361, 2001.

[64] M. Tehei and G. Zaccai, "Adaptation to extreme environments: macromolecular dynamics in complex systems," Biochimica et Biophysica Acta, vol. 1724, no. 3, pp. 404-410, 2005. 

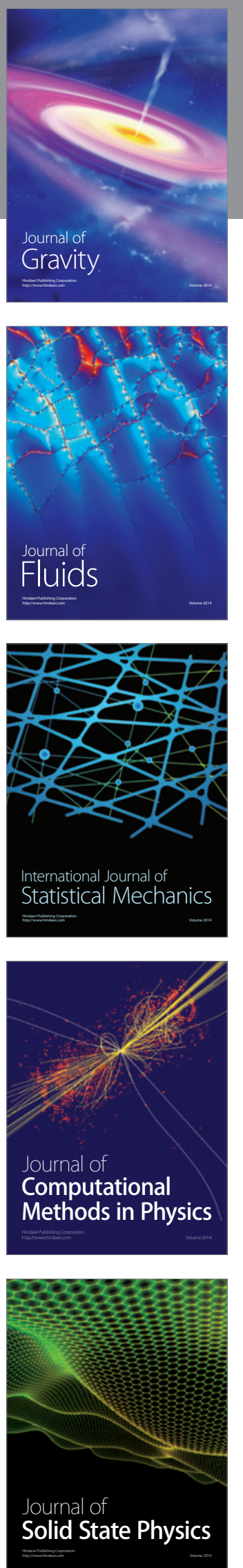

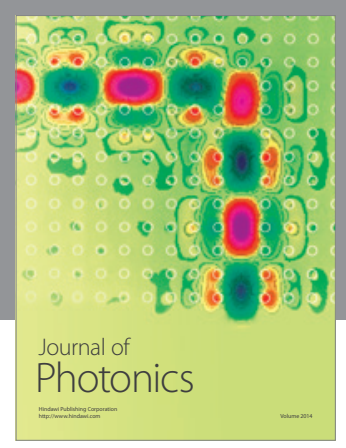

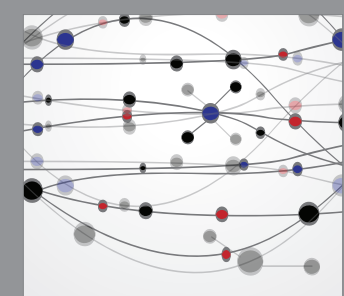

The Scientific World Journal
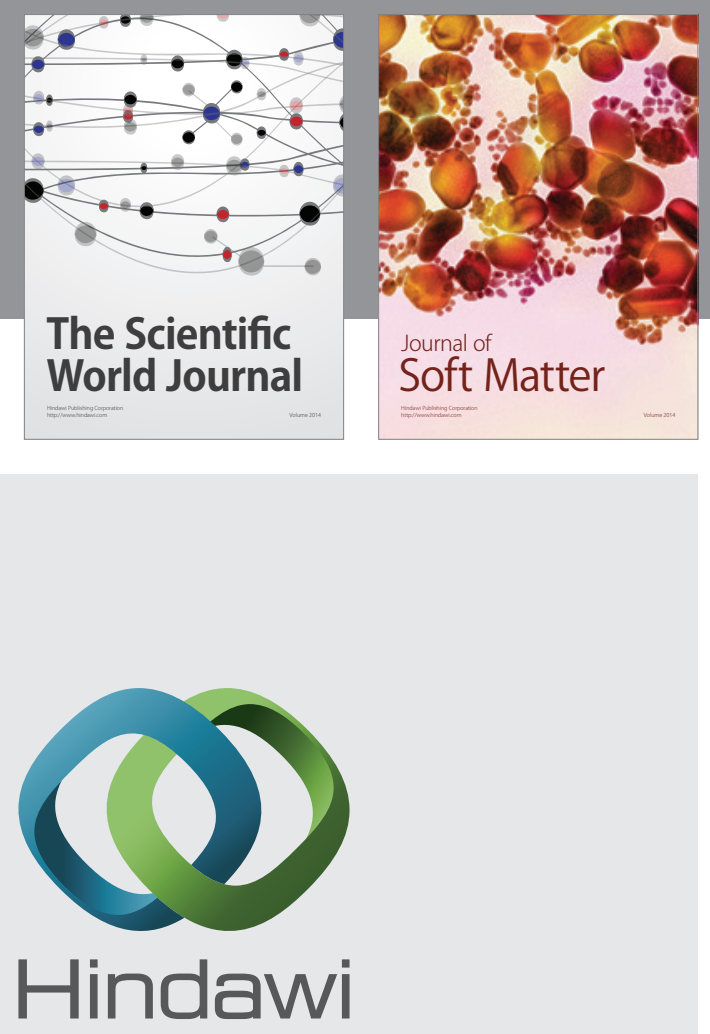

Submit your manuscripts at

http://www.hindawi.com
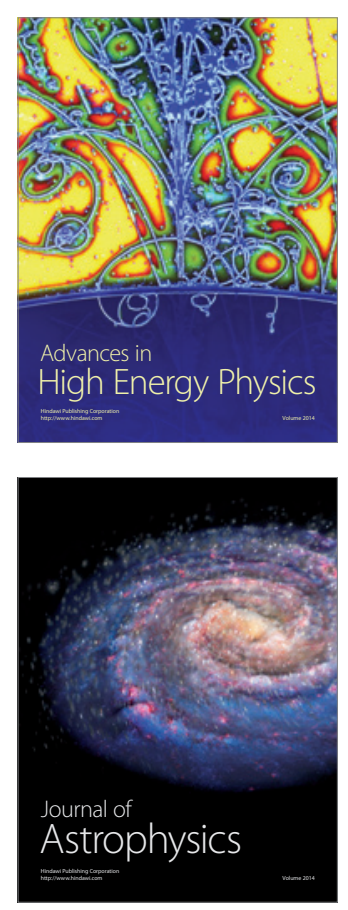
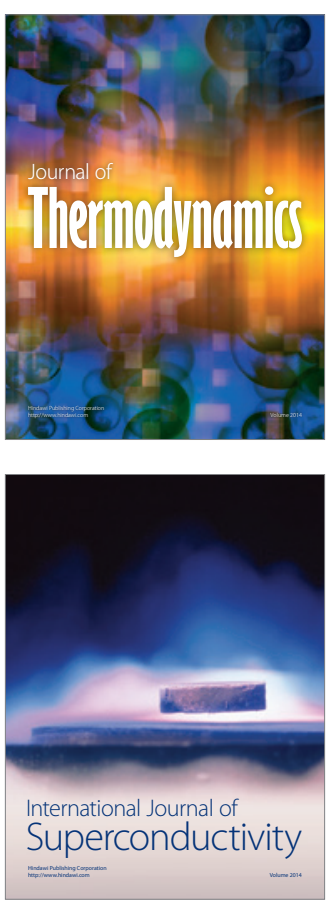
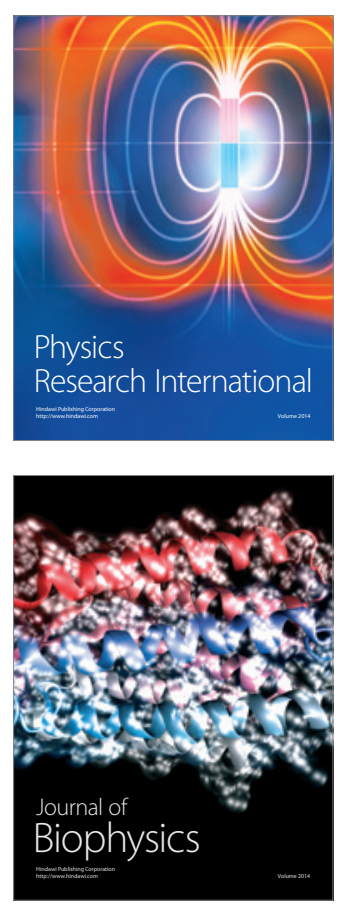
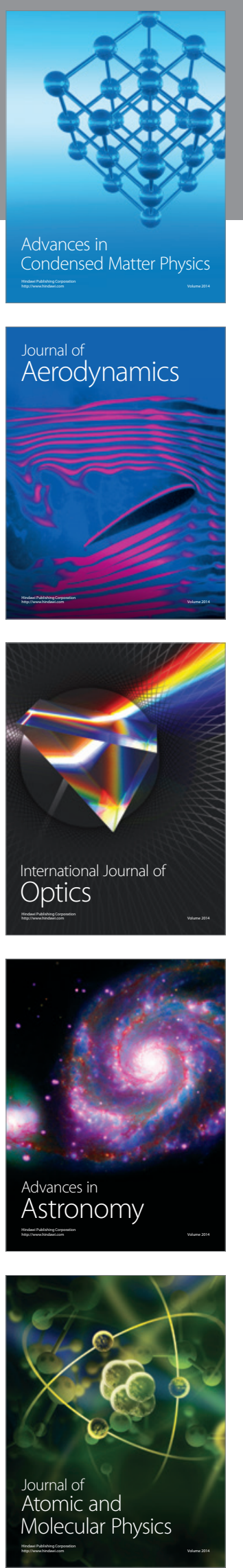